\title{
Análisis de redes sociales de la información sociobibliométrica
}

\author{
Jorge Ricardo Vivas
}

\begin{abstract}
Resumen
El presente trabajo examina los beneficios de utilizar Análisis de Redes Sociales en el campo de la exploración sociobibliométrica. Se consideran los límites y alcances prácticos y conceptuales. La propuesta es ilustrada utilizando un estudio realizado por Peiró y Carpintero (1981) sobre una red de revistas de modificación de la conducta. En este contexto se muestra la utilidad de usar las propiedades reticulares de Densidad, Centralidad, Intermediación, Poder y Agrupamiento como indicadores que permiten obtener información novedosa y complementaria a la extraída por los métodos clásicos de exploración bibliométrica.

Palabras Clave: Análisis de redes sociales; Sociobibliometría; Redes sociales.
\end{abstract}

\section{Social network analysis to cluster sociobibliometric information}

\begin{abstract}
This paper examines the benefits of using Social Network Analysis in the field of sociobibliometric exploration. There are considered practical and conceptual limits and reaches. The proposal is illustrated through a study about a journals network of behavior modification by Peiró and Carpintero (1981). In this context it is shown the utility of using reticular properties of Density, Centrality, Betweenness, Power and Clusterig as indicators that allow obtaining novel and complementary information to the one extracted by the classic methods of bibliometric exploration.
\end{abstract}

Keywords: Social network analysis; Sociobibliometry; Social networks.

El análisis de redes sociales conforma un núcleo conceptual y un arsenal metodológico que ha despertado un creciente interés en las dos últimas décadas (Degenne \& Forsé, 1994; Wasserman \& Faust, 1998; Wellman, 1999). Los investigadores de las ciencias sociales y del comportamiento, así como los metodólogos y los interesados en las ciencias de la administración y la información, hallaron en esta perspectiva una manera novedosa para formular y resolver preguntas clásicas de la investigación social. Una doble circunstancia ha colaborado en la propagación de esta inquietud; por un lado, el vertiginoso incremento de la disponibilidad informática para la aplicación de tratamientos numéricos complejos, largos y tediosos, de herramientas conceptuales ya concebidas a mediados de siglo. Por otro, la posibilidad de dar definición formal precisa a diferentes aspectos de la vida política, económica y social, centrando el análisis en las relaciones entre las entidades sociales, observando sus ritmos y regulaciones y describiendo estructuras a partir de la redundancia de esos patrones o regularidades.

Los estudios bibliométricos, por su parte, han sido definidos tradicionalmente como la cuantificación de la información bibliográfica que resulta susceptible de ser analizada (Garfield, Malin \& Small, 1978) y como la utilización de técnicas sociobibliométricas para el estudio cuantitativo y el análisis cualitativo de aquellas dimensiones de los materiales bibliográficos que permiten su medición (Carpintero \& Tortosa, 1990). Para comprender la multiplicidad de la producción académica se ha propuesto abordar a la ciencia como una organización, de modo de tener una visión coherente que conjugue la dimensión cualitativa, teórica y conceptual, y la dimensión cuantitativa, material y social (Carpintero 1981, 1998). Es a partir de la caracterización de la ciencia como organización que el estudio de la comunicación científica adquiere el status de estudio sociológico (López López, 1996) y permite aplicar, para comprender sus propiedades reticulares y estructura, las técnicas del análisis de redes sociales.

El concepto de estructura ha jugado un rol importante en los estudios sociales desde hace bastante tiempo. Lo que el análisis de redes sociales proporciona como novedad es la oferta de herramientas que permiten la operacionalización de este concepto, de modo tal que ahora es posible su análisis sistemático. Según Wellman (1988), el análisis de redes sociales difiere de los estudios tradicionales en que mientras éstos se concentran en el estudio de atributos individuales la primera estudia las relaciones sociales que los vinculan. Borgatti y Everett (1996) avanzan en esta idea al afirmar que, mientras las técnicas tradicionales mensuran atributos que afectan a los individuos y se denominan monádicos, el análisis de

Endereço para correspondência:

Funes 3.580 (7600) Mar del Plata

Tel/fax: (0223) 4752266

E-mail: jvivas@mdp.edu.ar 
redes estudia atributos diádicos que afectan a pares de individuos. Las relaciones sociales se expresan formalmente como atributos diádicos. Mientras la matriz del análisis clásico compone individuos con atributos, el análisis de redes usa una matriz que vincula individuos entre sí por medio de atributos diádicos.

Las relaciones sociales que pueden ser tratadas por el análisis de redes sociales aceptan una diversidad que contempla desde las interacciones amistosas de una pareja hasta el intercambio entre naciones. Lo importante es que a los fines del análisis ya no se trata de individuos que poseen cierto atributo, sino de individuos que se encuentran relacionados por compartir o no y en cierto grado, algunos atributos. El estudioso, entonces, se pregunta por la clase de intercambios, tangibles o no, que cohesionan una comunidad, por la emergencia de formas de agrupamiento por medio de ciertos lazos y relaciones, el flujo y la distancia comunicacional entre miembros de una organización o el intercambio de recursos o información en una comunidad científica.

El estudio de la ciencia como organización implica el estudio de su red comunicacional, toda vez que ésta es inherente a la existencia misma de la organización (Simon, 1964). La comunicación es el medio que permite el proceso de corrección, discusión $y$, eventualmente, la inclusión de los resultados obtenidos en el patrimonio intelectual de una comunidad científica relevante (Price, 1978). Se ha sostenido que su estudio es escencial para descubrir la estructural organizacional de la ciencia, en particular de la Psicología y se ha estimulado la aplicación de técnicas sociobibliométricas para lograr una aproximación contextualizada de la ciencia (Carpintero, 1981).

Lo que en este trabajo se sostiene es la utilidad de la utilización del análisis de redes sociales como un instrumento adecuado para un abordaje complementario al de las técnicas bibliométricas tradicionales. Su utilidad reside en su potencia para facilitar el análisis estructural de las relaciones sociales. Se presentan a continuación conceptos sustantivos del análisis de redes sociales y su vinculación con aspectos relevantes de la teoría y práctica sociobibliométrica.

\section{Relaciones, lazos y redes sociales}

Una red social es un conjunto de entidades sociales (personas u organizaciones) conectado por un conjunto de relaciones sociales. La red de comunicaciones en una comunidad científica constituye un caso particular de red social. Desde las investigaciones experimentales iniciales realizadas por Alex Bavelas $(1948,1950)$, se sostiene que la configuración de la red de comunicación determina el tipo de organización del grupo y sesga todos los comportamientos de sus participantes. El tipo de red afecta el comportamiento de sus miembros en diversos aspectos: su productividad, nivel de satisfacción, actividad global y roles quedan fuertemente condicionados por las propiedades reticulares de la comunicación (Mucchielli, 1991).

\section{Relaciones}

En el análisis de redes sociales la unidad de análisis es la relación social. Así como cables y ondas portadoras vinculan aparatos telefónicos, gráficamente la relación social es visualizada como una cuerda entre nodos, expresado esto en los términos propios del análisis de redes sociales. Una perspectiva mas formal, a su vez, la define como un vector que se constituye a partir de las siguientes dimensiones de la relación: contenido, dirección e intensidad.

El contenido se refiere a la clase de recurso que se esta intercambiando y mas específicamente, a algún atributo particular de la relación social a la que se hace referencia. Todas las mensuras que se realizan permiten reunir datos que sirven para caracterizar a una relación social, pero esas medidas no son la relación misma. Por ejemplo, la referencia bibliográfica es un aspecto mensurable de la relación académica entre investigadores y revistas, pero también es posible, y a veces necesario, considerar otros aspectos de la relación, como podría ser el período en el que se produjo, las presentaciones en coautoría, el momento en que comienzaron o las razones vinculares de su mantenimiento.

Las relaciones pueden o no tener una dirección. Por ejemplo, las referencias bibliográficas. Hay allí dos direcciones, emitir y recibir citas. En otros casos, como las relaciones fraternas, no existe direccionalidad por la naturaleza de la relación misma. Sin embargo, aún en el caso de compartir una relación inicialmente no direccionada, como puede ser la amistad, si bien en ciertos momentos puede que la relación sea simétrica, en otros es posible que encuentre una expresión asimétrica, en la medida que un actor exija una amistad mas íntima y otro una amistad mas débil, o uno de ambos ser el que mas frecuentemente busca al otro (Krackhardt, 1990).

Las relaciones también quedan caracterizadas por su intensidad. En algunas relaciones la fuerza del vínculo es fácilmente operacionalizada como en el análisis de redes de citas y referencias en una red de revistas, a través de la frecuencia y/o el volumen de información intercambiado. En otras relaciones la intensidad es de mas difícil captura. En esa misma red de revistas seguramente existe también un flujo de comunicación informal y la intensidad de estas relaciones puede requerir de medidas indirectas.

Lazos

Un lazo conecta un par de actores por medio de Psico-USF, v. 9, n. 1, p. 77-85, Jan./Jun. 2004 
una o mas relaciones. Un lazo se puede mantener sobre una sola relación, como ser miembro de una Sociedad Científica, o tener base múltiple asentada en diversas relaciones, como la de algunos miembros de la misma Sociedad pero que además comparten proyectos conjuntos, becarios, lengua y proximidad geográfica. Un lazo, en tanto conjunto de relaciones, también puede ser caracterizado por su contenido, dirección e intensidad. Esta última propiedad ha sido profusamente estudiada investigando los efectos sociales de los lazos fuertes y débiles. Granovetter (1973) ha sugerido la fortaleza de los vínculos débiles señalando que permiten integrar los subgrupos locales en conjuntos sociales mas amplios. Los miembros que comparten lazos mas fuertes incluyen normalmente mayor cantidad y calidad de relaciones (vecinos, simpatizantes de un mismo club, que envían sus hijos al mismo colegio, que comparten el financiamiento de servicios comunes, que se vinculan comercial y amistosamente, que practican deportes similares, que presentan relaciones familiares y noviazgos entre vecinos, etc.). Estos miembros, que probablemente estén mejor dispuestos a compartir los recursos propios con otros miembros del mismo grupo, se encuentran restringidos, sin embargo, por la limitación propia de la disponibilidad de los recursos existentes en ese grupo. Los lazos débiles, por el contrario, con portadores quizá menos dispuestos a compartirlos, proporcionan sin embargo acceso a recursos mas diversos, mas ricos y variados, ya que cada miembro pertenece, opera y tiene acceso a redes sociales que disponen de recursos diferentes.

En términos generales los analistas han encontrado que lazos mas fuertes, basados en relaciones múltiples, generan redes sociales mas cohesionadas, mas íntimas, mas densas y durables. Los lazos débiles, por el contrario, difícilmente se mantienen en el tiempo, pero facilitan la apertura que enriquece los grupos y permite su inclusión no intrusiva en conjuntos sociales mas amplios. La exploración de los lazos débiles en una comunidad científica por medio de las referencias bibliográficas, permite un estudio diferencial de la integración o segregación de lo distinto frente al paradigma dominante.

\section{Redes Sociales}

Una red social consiste en un conjunto finito de actores y de relaciones definidas entre ellos. Al estudiar las relaciones se distinguen dos tipos de variables que pueden ser incluidas como datos en una red: Variables estructurales y de composición (Wasserman \& Faust, 1998). Las primeras son tomadas para pares de actores y son las prototípicas del análisis de redes sociales. Las variables estructurales mensuran vínculos específicos de a pares de actores en una matriz, como pueden ser las citas bibliográficas, el intercambio comercial o las comunicaciones en una organización. Las variables de composición son datos de atributos de actor, se definen para cada actor individualmente y son las que se utilizan en la investigación psicológica y social estándar, como son datos de género, raza, C. I., desempeño, etc.

El estudio de los actores incluidos en una red es referido como el modo que adquiere la matriz según los distintos conjuntos de actores que son vinculados por variables estructurales. Cuando la misma variable (por ejemplo las citas) es medida para el mismo conjunto de actores o entidades sociales (por ejemplo las revistas especializadas en modificación de la conducta) se dice que estamos en presencia de una red de modo-uno. En otros estudios, donde se toma un conjunto de actores como emisores de una variable, por ejemplo conjunto de revistas de Psicología Cognitiva, y es tomado otro conjunto distinto como receptores de la misma, por ejemplo el conjunto de revistas de Neuropsicología, se refiere como redes modo-dos, ya que se trata con dos conjuntos de actores distintos. Borgatti y Everett (1996) señalan distintas alternativas para aplicar e interpretar las técnicas tradicionales de análisis de redes a redes modo-dos proponiendo, al mismo tiempo, el desarrollo de técnicas novedosas.

Cualquier red social puede ser considerada desde dos perspectivas diferentes; desde el punto de vista centrado en un individuo o mirando la red como un todo. La red ego-centrada parte de un actor local (denominado ego) y examina los lazos existentes en el conjunto de alteres vinculados con el ego y entre sí. Wellman y Wortley (1990), por ejemplo, utilizaron el análisis de redes ego-centradas para explorar los lazos que preservan el sentido de pertenencia a una comunidad mas allá de la proximidad geográfica. Las redes enteras, en cambio, se basan en algún criterio de delimitación de la población sobre el universo. Por ejemplo, el recorte podría remitirse a la red de revistas de modificación de la conducta, a los miembros de una organización, de un club o a los simpatizantes de un partido político. En este caso es objeto de tratamiento la totalidad de los lazos que se establecen entre los miembros de la red, la existencia o no del contacto, su frecuencia, la densidad del entramado social, las configuración de grupos y los roles y posiciones ocupados por los distintos miembros o subgrupos de miembros. $\mathrm{El}$ análisis de las redes personales permite identificar su tamaño y heternogeneidad, la calidad y cantidad de información y recursos a los que ese ego puede acceder. $\mathrm{El}$ análisis de redes enteras permite identificar miembros o grupos periféricos, su conectividad y la emergencia de miembros centrales y de otros que, sin serlo, actúan como intermediadores entre otros miembros de la red. 


\section{Propiedades Reticulares}

Resulta ilustrativa para esta presentación la selección de propiedades reticulares sugerida por Garton, Haythornthwaite y Wellman (1999) para el estudio de redes sociales en línea. Ellas son: rango, densidad, centralidad, roles y agrupamientos.

Rango. Esta propiedad alude al tamaño y a la diversidad. El rango se define como el número de miembros con los que tiene comunicación un miembro dado. Habitualmente hace referencia a vínculos simétricos, en los que no se considera la dirección. Cuando este atributo de la relación es importante, como en un estudio bibliométrico donde la dirección de la referencia es un dato significativo, se denomina rango de salida a la medida que considera los vínculos que parten de un miembro hacia los otros. La cantidad de referencias bibliográficas emitidas por un revista se puede mensurar a través de su Grado de Centralidad. En cambio, se habla de rango de entrada cuando se consideran sólo los vínculos que llegan a ella desde otros miembros de la misma red. Las citas bibliográficas pueden ser consideradas a través de la medida reticular de Prestigio en una red. Los rangos de salida y de entrada también se denominan redes de salida y de entrada, respectivamente.

Densidad. Esta es un atributo de la red que alude al volumen de interconexión entre sus miembros. De algún modo representa una medida de la cohesión de una red, que puede ser calculada también a través del estudio de las distancias geodésicas que separan a sus miembros. La densidad se mensura corrientemente como la proporción de conexiones efectivamente existentes sobre la totalidad de conexiones posibles en una red. Este número potencial de comunicaciones simétricas en una red de tamaño $\mathrm{N}$ se calcula como $\mathrm{N}(\mathrm{N}-1) / 2$, siendo que se habla de comunicación simétrica cuando no se considera el sentido del flujo comunicacional. En el estudio de la comunicación científica esta mensura permite tanto identificar subgrupos cohesivos dentro de una red mas amplia como la concentración de vínculos en la red personal de una revista, autor o grupo de investigación.

Centralidad. Es una de las propiedades mas intensivamente estudiada. En forma genérica revela, usando el volumen de conexiones para cada miembro de la red, la existencia de miembros que ocupan posiciones que varían en el grado de centralidad o periferia. En esta medida el análisis de redes sociales pone de manifiesto el legado moreniano ya que, en esencia, la mensura de tales atributos es propia de una matriz sociométrica. Un autor adquiere Prominencia en la medida que sus vínculos lo tornan particularmente visible para otros autores en la red.
Knoke y Burt (1983) distinguen dos tipos de Prominencia: Centralidad y Prestigio. Esta diferenciación solo es aplicable a relaciones direccionales, ya que el Prestigio es definido por los vínculos que llegan al actor, de modo que su valor crece en la medida en que el actor es objeto de más elecciones. El prestigio ha sido llamado Status por otros autores como Moreno (1934), Katz (1953) o Harary (1959). También hay medidas de centralidad basadas en la función de nexo o puente que un miembro puede desempeñar con relación a otros dos miembros en la red. La intermediación es un atributo que indica centralidad, posición de un miembro entre otros dos así conectados a través del primero, lo que representa consecuentemente, que éste potencialmente puede tener algún poder y control sobre las interacciones de los miembros no adyacentes. La fuerza de la intermediación disminuye en la medida en que los otros dos miembros conectados sean socialmente homogéneos y fuertemente conectados a través de otros posibles miembros intermedios. Esta es una propiedad que se puede encontrar en algunas revistas que funcionan como puente de divulgación cuando hay barreras idiomáticas. También se suele medir la pertenencia a un núcleo central por parte de un miembro como producto de su vinculación con pocos miembros pero muy centrales. Esta medida de centralidad se denomina habitualmente Poder y se suele presentar con revistas muy especializadas y prestigiosas pero reticentes a ofrecer sus referencias mas allá de los límites de un pequeño subgrupo de la red.

Agrupamientos. El agrupamiento es un indicador que permite diferenciar los miembros que están fuertemente conectados entre sí de los que no lo están. De hecho, constituye una estructura empíricamente descubierta por medio del estudio de las regularidades de patrones de interconexión entre los miembros de una red. La estructura y conformación de los subgrupos se obtiene a través del estudio de tres propiedades: ellos poseen un muy alta densidad (la mayoría de los lazos posibles efectivamente existen); la fuerza de la conexión entre miembros es elevada (medida en frecuencia, tiempo, elecciones, etc.); se encuentran herméticamente encapsulados, es decir, la mayoría de los lazos de salida caen en individuos del mismo conjunto. Para los analistas de redes sociales es importante saber que tipos y que patrones de relación definen la existencia de semejantes grupos y colaboran a su mantenimiento. A los agrupamientos escolásticos y disciplinares clásicos se agrega en la actualidad el estudio de la comunicación científica por medio de Listas académicas en Internet.

Roles y Posiciones. Las similitudes en los patrones de relación entre miembros diversos de un red o la similitud de patrones entre miembros de redes diferentes, sugiere la 
presencia de un rol en la red, ejercido desde una posición. Las regularidades son estudiadas como equivalencia estructural y homomorfismo y, en la práctica, permite visualizar como los miembros en posiciones semejantes acceden a recursos informativos similares, la forma en que algunos miembros ocupan posiciones que les permiten acceder a recursos de otras fuentes y aún de otras redes, y como otros miembros, en cambio, tienen acceso limitado a recursos fuera de su grupo. Es posible modelar bloques que identifiquen miembros que se encuentren en una posición estructural bibliométrica semejante, por ejemplo, de citar sin ser citados o ser citados por ciertos miembros y no por otros y referenciar a cierto grupo y no a otro. En general, la comparación de la estructura de roles en estudios bibliométricos puede permitir: describir miembros con rasgos similares en la misma red y/o grados de similitud entre redes diferentes.

\section{Aplicación de las propiedades reticulares al estudio bibliométrico \\ Para quienes están familiarizados con los} trabajos sobre colaboración científica el estudio de las relaciones entre autores establecidas por medio de la firma conjunta de publicaciones no constituye una novedad. Este método, propuesto por Price y Beaver (1966), ha permitido extraer núcleos de colaboración considerados "colegios invisibles" en los más diversos ámbitos del quehacer científico. El estudio del agrupamiento de autores según este indicador de colaboración constituye un entramado que se articula y delimita dentro de una red más amplia de los autores y publicaciones de la especialidad.

Partiendo de una matriz de referencias cruzadas de citas de autor el análisis de redes sociales proporciona medios prácticos y sencillos para seleccionar entre los métodos disponibles aquellos que mejor se ajusten a las necesidades del investigador. Por medio del análisis de la propiedad de agrupamiento en función de la proximidad y densidad entre actores es posible visualizar la conformación de subgrupos, su tamaño relativo, cohesión interna y las características de las relaciones que los vinculan intra e inter grupalmente. En el mismo sentido, es posible complementar los estudios de "Impacto" o "Visibilidad" de un autor o una revista que se obtienen con el método clásico de recontar las citas que su obra ha recibido en la literatura científica en un período determinado (Garfield et al., 1978). Knoke y Burt (1983) utilizando análisis de redes sociales equipararon el concepto de Prominencia con el de Visibilidad, desde que definieron la Prominencia de un autor como producto de la visibilidad obtenida por medio de los vínculos que un actor particular mantiene con cualquier otro de la red, pero considerando no solamente los vínculos directos con miembros adyacentes, sino también las vías indirectas a través de miembros intermediarios. De este modo, el análisis de redes sociales permite obtener otras medidas de impacto más sofisticadas, tanto por medio de los cuatro índices de Centralidad - Grado, Proximidad, Intermediación e Información - como a través del estudio del conjunto de las distancias geodésicas entre todos los miembros de la red.

A modo de ejemplo de cómo el estudio de las propiedades reticulares puede contribuir en el incremento de la capacidad explicativa de las medidas bibliométricas, tomaremos como ilustración una red de 25 revistas de modificación de la conducta seleccionadas para su estudio por Peiró y Carpintero (1981). La Matriz 1a ilustra la frecuencia de citas cruzadas, la Matriz 1b muestra la red de distancias e intermediación para ese mismo grupo de revistas. Siendo 1 el valor de la distancia mínima del camino óptimo entre dos revistas, se puede observar las fluctuaciones de los valores para cada una, tanto para cuando se totalizan las distancias desde una revista particular a cualquier otra de la red, como cuando se totalizan las distancias hacia una revista particular desde cualquiera otra revista de la red. Merece destacarse que la observación de esta matriz de distancias muestra cómo aún en aquellos casos en que no hay vínculo directo entre dos actores aparecen, sin embargo, los valores de distancia a que se encuentran en función de estar intermediados por otros actores igualmente vinculados con ambos. Así, los valores 2, 3, etcétera, expresan la distancia entre revistas cuando el vínculo depende de terceros actores a las que ambas se encuentran relacionadas en forma directa o indirecta.

Las Tablas 1 a y b, a continuación, muestran un núcleo de revistas extraídas por su valor máximo y mínimo. El subgrupo de revistas que poseen la mínima distancia a cualquier otra en la red constituyen el centro del grafo, son las que presentan mayor visibilidad y este grupo se denomina Centro Jordan, en memoria de su autor (Jordan, 1869).

Tabla 1a - Revistas que presentan $\Sigma$ menores distancias (Centro Jordan)
A cada otra revista
Desde cada otra revista
1) 21. PBUL (26)
1) 21. PBUL (25)
2) 13. JAPS (28)
2) 13. JAPS (26)
3) 22. PREP (28)
3) 4. AMPS (29)
4) 8. BTHE (28)
4) 15. JCCP (29)
5) 4. AMPS (29)
5) 7. BRTH (31)

Psico-USF, v. 9, n. 1, p. 77-85, Jan./Jun. 2004 
Tabla $1 \mathrm{~b}$ - Revistas que presentan $\Sigma$ mayores distancias (Periferia)

\begin{tabular}{lll}
\hline A cada otra revista & & \multicolumn{2}{c}{ Desde cada otra revista } \\
1) $17 . \mathrm{JEAB} \mathrm{(45)}$ & & 1) 20. MENT (52) \\
2) 9. BEHA (45) & - & 2) 6. BRMI (48) \\
3) 23. PREV (44) & 3) 9. BEHA (47) \\
4) 20. MENT (42) & 4) 12. JACH (45) \\
5) 25. PMED (41) & - & 5) 17. JEAB (40) \\
\hline
\end{tabular}

La Figura 1, complementariamente, nos muestra como en función del análisis de la densidad, fortaleza, dirección y autoalimentación de la red de revistas considerada, se pueden separar para su estudio 18 subgrupos que cumplen con estas condiciones.

\begin{tabular}{|c|c|c|c|c|c|c|c|c|c|c|c|c|c|c|c|c|c|c|c|c|c|c|c|c|c|}
\hline & & & & 1 & 2 & 2 & & 1 & 1 & & 1 & 1 & 1 & & 1 & & 1 & & & 1 & 1 & 2 & 2 & 2 & 2 \\
\hline Level & 1 & 6 & 9 & 7 & 0 & 3 & 2 & 1 & 6 & 3 & 0 & 2 & 4 & 5 & 9 & 4 & 8 & 7 & 8 & 5 & 3 & 1 & 2 & 4 & 5 \\
\hline -------- & - & - & - & - & - & - & - & - & - & - & - & - & - & - & - & - & - & - & - & - & - & - & - & - & - \\
\hline 18 & . & . & . & . & . & . & . & . & . & . & . & . & . & . & . & . & . & . & . & \multicolumn{4}{|c|}{ XXXXXXX } & . & . \\
\hline 16 & . & . & . & . & . & . & . & . & . & . & . & . & . & . & . & . & . & . & & \multicolumn{4}{|c|}{ XXXXXXXX } & . & . \\
\hline 14 & . & . & . & . & . & . & . & . & . & . & . & . & . & . & . & $\cdot$ & . & XX & \multicolumn{5}{|c|}{ XXXXXXXX } & . & . \\
\hline 11 & . & . & . & . & . & . & . & . & . & . & . & . & . & . & . & XX & $\mathrm{XX}$ & $x \times X$ & \multicolumn{5}{|c|}{ XXXXXXXXX } & . & . \\
\hline 10 & . & . & . & . & . & . & . & . & . & . & . & . & . & . & . & XX & $\mathrm{XX}$ & $x \times X$ & \multicolumn{6}{|c|}{ XXXXXXXXXXX } & . \\
\hline 9 & . & . & . & . & . & . & . & . & . & $\cdot$ & $\cdot$ & $\cdot$ & $\cdot$ & $\mathrm{XX}$ & XX & $\mathrm{XX}$ & $\mathrm{XX}$ & $x \times X$ & \multicolumn{5}{|c|}{ XXXXXXXXXXX } & & . \\
\hline 7 & . & . & . & . & . & . & . & . & . & XX & XX & $X X$ & $X X$ & XXX & $X Y$ & $\mathrm{XX}$ & $\mathrm{XX}$ & KXX & \multicolumn{6}{|c|}{ XXXXXXXXXX } & . \\
\hline 4 & . & . & . & . & . & . & . & . & . & $\mathrm{XX}$ & XX & $\mathrm{XX}$ & $X X$ & $\mathrm{XX}$ & $X Y$ & $\mathrm{XX}$ & $\mathrm{XX}$ & XX & \multicolumn{7}{|c|}{ XXXXXXXXXXXXX } \\
\hline 2 & $\cdot$ & 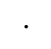 & . & & . & . & XY & & $\lambda \lambda$ & $X X$ & XX & $X X$ & $X X$ & $\mathrm{XX}$ & XY & $\mathrm{XX}$ & XX & $\mathrm{XX}$ & \multicolumn{7}{|c|}{ XXXXXXXXXXXXX } \\
\hline 0 & & $\mathrm{X}$ & $\mathrm{X}$ & & $\mathrm{XY}$ & XY & $\mathrm{XX}$ & & $X X$ & $\mathrm{XX}$ & XX & $\mathrm{XX}$ & $X X$ & XX & & $\mathrm{XX}$ & & $\mathrm{XX}$ & \multicolumn{7}{|c|}{ XXXXXXXXXXXX } \\
\hline
\end{tabular}

Figura 1 - Agrupamiento vinculado jerárquicamente

En el nivel más alto se puede observar como las revistas que conforman el Centro Jordan son miembros que integran la totalidad de las cliques hasta los niveles más bajos. En este caso las revistas son la 21 (Psychological Bulletin), la 22 (Psychological Reports), la 13 (Journal of Abnormal Psychology) y la 15 (Journal of Counseling and Clinical Psychology). Clique o pandilla es una denominación que debe su origen al estudio de las redes de pandillas callejeras. En la base del gráfico, complementariamente, se observan revistas que no pertenecen a ningún subgrupo y presentan el mayor nivel de periferia. Estas son 1 (American Journal of Mental Deficiency), 6 (Behavior Research Method and Instruments), 9 (Behaviorism), 17 (Journal of Experimental Analysis of Behavior), 20 (Mental Retardation) y 23 (Psychological Review).

\section{Consideraciones finales}

La utilización del núcleo conceptual del Análisis de Redes Sociales parece ser especialmente adecuada para integrarse con los estudios sociobibliométricos desde que para ambas perspectivas existen posiciones teóricas comunes y congruentes. Para ambas los actores sociales y sus actos son vistos como mutuamente interdependientes, los vínculos que los unen se conciben como canales de flujo de recursos materiales o inmateriales, las estructuras son vistas como patrones que indican regularidades en las relaciones entre actores y las redes a las que pertenecen son consideradas como proveedoras de oportunidades o restricciones a la acción individual de los actores.

Paralelamente, la batería metodológica del análisis de redes sociales puede contribuir sustantivamente con los estudios sociobibliométricos poniendo a su servicio herramientas de tratamiento de la información que faciliten la elucidación de distintos aspectos de su quehacer teórico y práctico. El análisis de redes sociales provee de numerosos instrumentos útiles para dar definición formal a conceptos teóricos, facilita la concreción de modelos formales, permite la evaluación y puesta a prueba de teorías, integra en un mismo entorno de trabajo herramientas estadísticas, de álgebra de matrices y de teoría de grafos y facilita, en ofertas desarrolladas para diferentes necesidades, una gran variedad de aplicaciones informáticas de libre acceso.

Desde esta perspectiva autores como Hiltz y Turoff (1993) pudieron aplicar herramientas del análisis de redes sociales al estudio de colegios invisibles. Por este medio les fue posible obtener una imagen más organi-zada y estructurada del campo, al visualizar subgrupos relevantes de colaboración y sus interrelaciones, la centralidad y prestigio de sus componentes y el tamaño y heterogeneidad de la red personal de los miembros más centrales del colegio. En el mismo sentido pueden interpretarse las antes mencionadas contribuciones de Knoke y Burt (1983) al estudio de la prominencia 
utilizando análisis de redes sociales. Su aplicación ha permitido avanzar en una caracterización más fina de la visibilidad, al considerar no sólo las medidas directas de la relación entre actores sino también las que surgen de la fortaleza relativa de la relación com otros agentes sociales que funcionan así como intermediarios de la visibilidad.

Para finalizar es oportuno recordar que el concepto de estructuras y redes ha tenido un profuso desarrollo en Psicología y Ciencias Sociales, aunque no, lamentablemente, el nivel de precisión semántica o rigurosidad formal, como para hacer de ellos una herramienta conceptual ni instrumental biunívoca compartida. Los diferentes obstáculos epistemológicos y sociales entre las disciplinas se vieron agravados por la falta de tecnología adecuada para facilitar las operaciones requeridas por los desarrollos teóricos. Hoy estas barreras van siendo superadas y el software actual permite la confección de matrices de datos y su posterior tratamiento con eficiencia y rapidez, en entornos computacionales amigables que tienen un alto grado de compatibilidad con los paquetes estadísticos usuales.

Por estas razones el presente trabajo, menos que la defensa de una prescripción metodológica, está imbuido de un espíritu anfitrión que desea modificar la impronta normativa por la sugerencia que invita a la experimentación por mano propia, que complementa y enriquece los tratamientos habituales y corrientes.

\section{Referencias}

Bavelas, A. (1948). A mathematical model for group structures. Human Organization, 7, 16-30.

Bavelas, A. (1950). Communications patterns in task oriented groups. Journal of the Acoustical Society of América, 22, 271-282.

Borgatti, S. \& Everett, M. (1996). Mode-2 data set network analysis. Journal of Computer Mediated Communication. 2 (1).

Carpintero, H. (1981). La psicología actual desde una perspectiva bibliométrica: una introducción. Em H. Carpintero \& J. M. Peiró (Dirs.). Psicología contemporánea. Teoría y métodos cuantitativos para el estudio de su literatura cientifica. Valencia: Ed. Alfaplus.

Carpintero, H. (1998). Introducción a las técnicas sociobibliométricas para el estudio de la literatura científica en Psicología. Curso de actualización. Mar del Plata: UNMDP.

Carpintero, H. \& Tortosa, F. (1990). Aplicaciones de la metodología bibliométrica a la historia de Psicología: una revisión de conjunto. Em F. Tortosa, L. Mayor \& H. Carpintero (Dirs.). La Psicología contemporánea desde la Historiografía. Barcelona: PPU.
Degenne, A. \& Forsé, M. (1994). Les reseaux sociaux. une analyse structurale en sociologie. Paris: Armand Colin.

Garfield, E., Malin, M. V. \& Small, H. (1978). Citation data as science indicators. Em Y. Elkana et al. Towar a metric of science. New York: Wiley.

Garton, L., Haythornthwaite, C. \& Wellman, B. (1999). Studying on-line social networks. Em Steve Jones (Ed.). Doing internet research. Thousand Oaks, CA: Sage.

Granovetter, M. (1973). The strength of weak ties. American Journal of Sociology, 78, 1360-1380.

Harary, F. (1959). Status and Contrastatus. Sociometry, $22,23-43$.

Hiltz, S. R. \& Turoff, M. (1993). The network nation. (second ed.). Cambridge, MA: MIT Press.

Jordan, C. (1869). Sur les assemblages de lignes. Journal für die reine und angewandte Mathematic, 70, 185-90.

Katz, L. (1953). A new status index derived from sociometric analysis. Psychometrika, 18, 39-43.

Knoke, D. \& Burt, R. S. (1983). Prominence. Em R. S. Burt y M. J. Minor (Eds.). Applied network analysis. Newbury Park, CA: Sage.

Krackhardt, D. (1990). Assessing the political landscape: structure, cognition and power in organizations. Administrative Science Quarterly, 35, 342-369.

López López, P. (1996). Introducción a la bibliometría. Valencia: Promolibro.

Moreno, J. L. (1934). Who shall survive? Fundations of sociometry, group psychotherapy and sociodrama. Washington, D.C.: Nervous and Mental Disease Publishing Co.

Mucchielli, R. (1991). Communication et reseaux de communications. Nice: ESF.

Peiró, M. \& Carpintero, H. (1981). Revistas en modificación de la conducta: un estudio de la red de comunicación en la especialidad. Em H. Carpintero \& J. M. Peiró (Dirs.). Psicología Contemporánea. Teoría y métodos cuantitativos para el estudio de su literatura cientifica. Valencia: Ed. Alfaplus.

Price, D. J. (1978). Toward a model for science indicators. Em I. Elkana et al. Toward a metric of science. New York: Wiley.

Price, D. J. \& Beaver, D. (1966). Collaboration in an invisible college. American Psychologist, 21, 1011-1018.

Simon, H. (1964). El comportamiento administrativo. Madrid: Aguilar.

Wasserman, S. \& Faust, K. (1998). Social network analysis. methods and applications. Cambridge: Cambridge University Press.

Wellman, B. (1988). Thinking structurally. Em Barry 
Wellman \& S. D. Berkowitz (Eds.). Social structures: A network approach. Cambridge: Cambridge University Press.

Wellman, B. (1999). Networks in the Global Village. CO:

Westview Press.
Wellman, B. \& Wortley, S. (1990). Different strokes from different folks: Community ties and social support. American Journal of Sociology, 81, 730-780.

Recebido em outubro de 2003

Reformulado em maio de 2004 Aprovado em junho de 2004

Matriz 1a: Frecuencias de citas emitidas y recibidas entre las 25 revistas estudiadas (Peiró y Carpintero, 1981)

\begin{tabular}{|c|c|c|c|c|c|c|c|c|c|c|c|c|c|c|c|c|c|c|c|c|c|c|c|c|c|c|}
\hline & 1 & 2 & 3 & 4 & 5 & 6 & 7 & 8 & 9 & 10 & 11 & 12 & 13 & 14 & 15 & 16 & 17 & 18 & 19 & 20 & 21 & 22 & 23 & 24 & 25 & Total \\
\hline 1 & 271 & 0 & 0 & 9 & 0 & 0 & 0 & 0 & 0 & 0 & 47 & 0 & 18 & 21 & 0 & 0 & 0 & 0 & 0 & 39 & 20 & 7 & 14 & 0 & 0 & \\
\hline 2 & 7 & 70 & 25 & 9 & 20 & 0 & 0 & 0 & 0 & 7 & 26 & 0 & 6 & 0 & 13 & 0 & 0 & 0 & 7 & 0 & 10 & 0 & 0 & 0 & 0 & \\
\hline 3 & 0 & 29 & 743 & 9 & 456 & 0 & 8 & 0 & 0 & 145 & 0 & 0 & 22 & 0 & 25 & 0 & 0 & 78 & 0 & 0 & 10 & 11 & 0 & 0 & 41 & 1577 \\
\hline 4 & 0 & 9 & 14 & 326 & 12 & 0 & 7 & 8 & 10 & 9 & 11 & 0 & 33 & 12 & 28 & 7 & 0 & 9 & 45 & 0 & 46 & 9 & 46 & 13 & 12 & 660 \\
\hline 5 & 0 & 21 & 361 & 14 & 509 & 0 & 12 & 15 & 0 & 167 & 0 & 0 & 29 & 0 & 57 & 12 & 0 & 86 & 0 & 0 & 29 & 26 & 0 & 8 & 8 & 1354 \\
\hline 6 & 0 & 0 & 0 & 19 & 7 & 197 & 0 & 0 & 0 & 0 & 0 & 0 & 6 & 0 & 7 & 6 & 33 & 0 & 0 & 0 & 22 & 0 & 21 & 44 & 11 & 373 \\
\hline 7 & 8 & 0 & 0 & 6 & 12 & 0 & 116 & 82 & 0 & 16 & 0 & 0 & 47 & 36 & 37 & 48 & 0 & 0 & 8 & 0 & 14 & 9 & 0 & 20 & 23 & 482 \\
\hline 8 & 8 & 0 & 12 & 26 & 23 & 0 & 127 & 212 & 9 & 22 & 22 & 0 & 68 & 127 & 115 & 56 & 15 & 9 & 27 & 6 & 32 & 23 & 14 & 0 & 12 & 965 \\
\hline 9 & 0 & 0 & 0 & 14 & 0 & 0 & 0 & 0 & 18 & 0 & 0 & 0 & 0 & 0 & 0 & 0 & 18 & 0 & 0 & 0 & 6 & 0 & 31 & 0 & 0 & \\
\hline 10 & 0 & 9 & 118 & 0 & 159 & 0 & 22 & 0 & 0 & 474 & 0 & 0 & 13 & 0 & 14 & 0 & 0 & 41 & 0 & 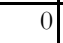 & 8 & 10 & 0 & 0 & 9 & 877 \\
\hline 11 & 10 & 17 & 0 & 13 & 0 & 0 & 0 & 0 & 0 & 0 & 399 & 0 & 26 & 0 & 21 & 0 & 0 & 0 & 82 & 0 & 62 & 13 & 37 & 6 & 0 & 686 \\
\hline 12 & 20 & 15 & 14 & 0 & 16 & 0 & 19 & 22 & 0 & 0 & 32 & 35 & 19 & 39 & 48 & 0 & 0 & 9 & 7 & 0 & 16 & 14 & 0 & 11 & 0 & 336 \\
\hline 13 & 6 & 9 & 21 & 15 & 63 & 0 & 23 & 8 & 0 & 36 & 7 & 11 & 272 & 19 & 60 & 0 & 13 & 44 & 89 & 0 & 49 & 37 & 12 & 13 & 6 & 813 \\
\hline 14 & 17 & $\overline{6}$ & 0 & 0 & 7 & 0 & 37 & 61 & 0 & 0 & 24 & 0 & 12 & 264 & 8 & 9 & 0 & 0 & 9 & 23 & 10 & 0 & 0 & 0 & 0 & 487 \\
\hline 15 & 0 & 10 & 35 & 52 & 55 & 0 & 86 & 108 & 0 & 22 & 15 & 8 & 216 & 36 & 615 & 27 & 0 & 7 & 0 & 0 & 10 & 11 & 0 & 0 & 0 & 1313 \\
\hline 16 & 7 & 0 & 6 & 9 & 12 & 0 & 47 & 51 & 0 & 8 & 0 & 0 & 19 & 50 & 27 & 60 & 0 & 7 & 0 & 0 & 10 & 11 & 0 & 0 & 0 & 324 \\
\hline 17 & 0 & $\overline{0}$ & 0 & 6 & 0 & 0 & 0 & 0 & 0 & 0 & 0 & 0 & 0 & 0 & 0 & 0 & 961 & 0 & 0 & 0 & 23 & 25 & 61 & 0 & 0 & 1076 \\
\hline 18 & 0 & 7 & 163 & 7 & 177 & 0 & 13 & 11 & 0 & 76 & 0 & 0 & 66 & 0 & 43 & 8 & 0 & 117 & 9 & 0 & 12 & 14 & 15 & 7 & 39 & 784 \\
\hline 19 & 0 & 0 & 6 & 48 & 0 & 0 & 6 & 9 & 9 & 9 & 24 & 0 & 128 & 0 & 45 & 0 & 0 & 6 & 682 & 0 & 96 & 10 & 87 & 17 & 9 & 1191 \\
\hline 20 & 53 & 0 & 0 & 0 & 0 & 0 & 12 & 4 & 0 & 0 & 4 & 0 & 4 & 25 & 0 & 0 & 4 & 0 & 0 & 71 & 0 & 0 & 0 & 0 & 0 & \\
\hline 21 & 22 & 21 & 63 & 49 & 94 & 11 & 80 & 94 & 0 & 54 & 53 & 17 & 155 & 47 & 142 & 47 & 36 & 34 & 232 & 0 & 204 & 66 & 74 & 28 & 30 & 1653 \\
\hline 22 & 12 & 10 & 50 & 30 & 42 & 0 & 33 & 37 & 0 & 12 & 101 & 0 & 135 & 20 & 222 & 15 & 34 & 21 & \begin{tabular}{|l|}
150 \\
\end{tabular} & 0 & \begin{tabular}{|l|}
115 \\
\end{tabular} & 263 & 55 & 9 & 13 & 1379 \\
\hline 23 & 0 & $\overline{0}$ & 0 & 16 & 0 & 0 & 0 & 0 & 0 & 0 & 0 & 0 & 9 & 0 & 0 & 0 & 36 & 0 & 21 & 0 & 19 & 0 & 126 & 0 & 0 & 227 \\
\hline 24 & 0 & $\overline{0}$ & 0 & 14 & 20 & 0 & 16 & 8 & 0 & 6 & 0 & 0 & 57 & 7 & 9 & 0 & 0 & 22 & 24 & 0 & 36 & 0 & 18 & \begin{tabular}{|l|}
293 \\
\end{tabular} & 26 & \\
\hline 25 & 0 & 0 & 14 & 0 & 48 & 0 & 8 & 0 & 0 & 7 & 0 & 0 & 15 & 0 & 15 & 0 & 0 & 7 & 0 & 0 & 6 & 0 & 0 & 24 & 122 & 260 \\
\hline Tot & 441 & 233 & \begin{tabular}{|l|}
1645 \\
\end{tabular} & 691 & 1732 & 208 & 672 & 730 & 46 & 1070 & 765 & 71 & 1375 & 703 & 1551 & 295 & 1150 & 497 & 1392 & 139 & 865 & 559 & 611 & 493 & 361 & 1829 \\
\hline
\end{tabular}


Matriz 1b - Matriz de Adyacencias correspondiente a la Matriz 1a

\begin{tabular}{|c|c|c|c|c|c|c|c|c|c|c|c|c|c|c|c|c|c|c|c|c|c|c|c|c|c|c|}
\hline & 1 & 2 & 3 & 4 & 5 & 6 & 7 & 8 & 9 & 10 & 11 & 12 & 13 & 14 & 15 & 16 & 17 & 18 & 19 & 20 & 21 & 22 & 23 & 24 & 25 & Total \\
\hline 1 & 0 & 2 & 2 & 1 & 2 & 2 & 2 & 2 & 2 & 2 & 1 & 2 & 1 & 1 & 2 & 2 & 2 & 2 & 2 & 1 & 1 & 1 & 1 & 2 & 2 & 40 \\
\hline 2 & 1 & 0 & 1 & 1 & 1 & 2 & 2 & 2 & 2 & 1 & 1 & 2 & 1 & 2 & 1 & 2 & 2 & 2 & 1 & 2 & 1 & 2 & & 2 & 2 & 38 \\
\hline 3 & 2 & 1 & 0 & 1 & 1 & 2 & 1 & 2 & 2 & 1 & 2 & 2 & 1 & 2 & 1 & 2 & 2 & 1 & 2 & 3 & 1 & 1 & 2 & 2 & 1 & 38 \\
\hline 4 & 2 & 1 & 1 & 0 & 1 & 2 & 1 & 1 & 1 & 1 & 1 & 2 & 1 & 1 & 1 & 1 & 2 & 1 & 1 & 2 & 1 & 1 & 1 & 1 & 1 & 29 \\
\hline 5 & 2 & 1 & 1 & 1 & 0 & 2 & 1 & 1 & 2 & 1 & 2 & 2 & 1 & 2 & 1 & 1 & 2 & 1 & 2 & 2 & 1 & 1 & 7 & 1 & 1 & 34 \\
\hline 6 & 2 & 2 & 2 & 1 & 1 & 0 & 2 & 2 & 2 & 2 & 2 & 2 & 1 & 2 & 1 & 1 & 1 & 2 & 2 & 3 & 1 & 2 & 1 & 1 & 1 & 39 \\
\hline 7 & 1 & 2 & 2 & 1 & 1 & 2 & 0 & 1 & 2 & 1 & 2 & 2 & 1 & 1 & 1 & 1 & 2 & 2 & 1 & 2 & 1 & 1 & 2 & 1 & 1 & 34 \\
\hline 8 & 1 & 2 & 1 & 1 & 1 & 2 & 1 & 0 & 1 & 1 & 1 & 2 & 1 & 1 & 1 & 1 & 1 & 1 & 1 & 1 & 1 & 1 & 1 & 2 & 1 & 28 \\
\hline 9 & 2 & 2 & 2 & 1 & 2 & 2 & 2 & 2 & 0 & 2 & 2 & 2 & 2 & 2 & 2 & 2 & 1 & 2 & 2 & 3 & 1 & 2 & 1 & 2 & 2 & 45 \\
\hline 10 & 2 & 1 & 1 & 2 & 1 & 2 & 1 & 2 & 3 & 0 & 2 & 2 & 1 & 2 & 1 & 2 & 2 & 1 & 2 & 3 & 1 & 1 & 3 & 2 & 1 & 40 \\
\hline 11 & 1 & 1 & 2 & 1 & 2 & 2 & 2 & 2 & 2 & 2 & 0 & 2 & 1 & 2 & 1 & 2 & 2 & 2 & 1 & 2 & 1 & 1 & 1 & 1 & 2 & 38 \\
\hline 12 & 1 & 1 & 1 & 2 & 1 & 2 & 1 & 1 & 2 & 2 & 1 & 0 & $\overline{1}$ & 1 & 1 & 2 & 2 & 1 & 1 & 2 & 1 & 1 & 2 & 1 & 2 & 33 \\
\hline 13 & 1 & 1 & 1 & 1 & 1 & 2 & 1 & 1 & 2 & 1 & 1 & 1 & 0 & 1 & 1 & 2 & 1 & 1 & 1 & 2 & 1 & 1 & 1 & 1 & 1 & 28 \\
\hline 14 & 1 & 1 & 2 & 2 & 1 & 2 & 1 & 1 & 2 & 2 & 1 & 2 & 1 & 0 & 1 & 1 & 2 & 2 & 1 & 1 & 1 & 2 & 2 & 2 & 2 & 36 \\
\hline 15 & 2 & 1 & 1 & 1 & 1 & 2 & 1 & 1 & 2 & 1 & 1 & 1 & 1 & 1 & 0 & 1 & 2 & 1 & 2 & 2 & 1 & 1 & 2 & 2 & 2 & 33 \\
\hline 16 & 1 & 2 & 1 & 1 & 1 & 2 & 1 & 1 & 2 & 1 & 2 & 2 & 1 & 1 & 1 & 0 & 2 & 1 & 2 & 2 & 1 & 1 & 2 & 2 & 2 & 35 \\
\hline 17 & 2 & 2 & 2 & 1 & 2 & 2 & 2 & 2 & 2 & 2 & 2 & 2 & 2 & 2 & 2 & 2 & 0 & 2 & 2 & 3 & 1 & 1 & 1 & 2 & 2 & 45 \\
\hline 18 & 2 & 1 & 1 & 1 & 1 & 2 & 1 & 1 & 2 & 1 & 2 & 2 & 1 & 2 & 1 & 1 & 2 & 0 & 1 & 2 & 1 & 1 & 1 & 1 & 1 & 32 \\
\hline 19 & 2 & 2 & 1 & 1 & 2 & 2 & 1 & 1 & 1 & 1 & 1 & 2 & 1 & 2 & 1 & 2 & 2 & 1 & 0 & 2 & 1 & 1 & 1 & 1 & 1 & 33 \\
\hline 20 & 1 & 2 & 2 & 2 & 2 & 3 & 1 & 1 & 2 & 2 & 1 & 2 & 1 & 1 & 2 & 2 & 1 & 2 & 2 & 0 & 2 & 2 & 2 & 2 & 2 & 42 \\
\hline 21 & 1 & 1 & 1 & 1 & 1 & 1 & 1 & 1 & 2 & 1 & 1 & 1 & 1 & 1 & 1 & 1 & 1 & 1 & 1 & 2 & 0 & 1 & 1 & 1 & 1 & 26 \\
\hline 22 & 1 & 1 & 1 & 1 & 1 & 2 & 1 & 1 & 2 & 1 & 1 & 2 & 1 & 1 & 1 & 1 & 1 & 1 & 1 & 2 & 1 & 0 & 1 & 1 & 1 & 28 \\
\hline 23 & 2 & 2 & 2 & 1 & 2 & 2 & 2 & 2 & 2 & 2 & 2 & 2 & 1 & 2 & 2 & 2 & 1 & 2 & 1 & 3 & 1 & 2 & 0 & 2 & 2 & 44 \\
\hline 24 & 2 & 2 & 2 & 1 & 1 & 2 & 1 & 1 & 2 & 1 & 2 & 2 & 1 & 1 & 1 & 2 & 2 & 1 & 1 & 2 & 1 & 2 & 1 & 0 & 1 & 35 \\
\hline 25 & 2 & 2 & 1 & 2 & 1 & 2 & 1 & 2 & 3 & 1 & 2 & 2 & 1 & 2 & 1 & 2 & 2 & 1 & 2 & 3 & 1 & 2 & 2 & 1 & 0 & 41 \\
\hline ot & 37 & 36 & 34 & 29 & 31 & 48 & 31 & 34 & 47 & 33 & 36 & 45 & 26 & 36 & 29 & 38 & 40 & 34 & 35 & 52 & 25 & 32 & 35 & 36 & 35 & 894 \\
\hline
\end{tabular}

Sobre o autor:

Jorge Ricardo Vivas é psicólogo, mestre em Psicologia Social, professor titular e coordenador do Grupo de Investigación en Psicología Cognitiva y Educacional na Facultad de Psicología da Universidad Nacional de Mar del Plata. 
